East African Medical Journal Vol. 81 No. 4 April 2004

DEATH FROM BODY PACKER SYNDROME: CASE REPORT

A. K. O., Olumbe, MBChB (Path), DMJ (Path), Dip. Forens. Med. (RCPA), Former Chief Government, Pathologist of Kenya, Ministry of Health, and A.Y. Kalebi, MBChB, Registrar, Department of Human Pathology, College of Health Sciences, University of Nairobi, P.O. Box 19676, Nairobi, Kenya

Request for reprints to: Dr. A.Y. Kalebi, Department of Human Pathology College of Health Sciences, University of Nairobi, P.O Box 19676, Nairobi, Kenya

\title{
DEATH FROM BODY PACKER SYNDROME: CASE REPORT
}

\author{
A. K. O. OLUMBE and A.Y. KALEBI
}

\begin{abstract}
We report a case of death due to the effects of heroin concealed in a woman who was attempting to smuggle the drug into Kenya concealed within her gastro-intestinal tract. She was arrested at a Nairobi airport. While under police detention, she expelled a pellet par anum containing the drug then collapsed, after being forcefully fed on a heavy meal. She was taken to hospital in coma where she expelled nine pellets and died three days later. Her blood level of heroin at admission was well beyond the lethal dose. Details are provided of the circumstances leading to her admission into hospital, her stay in hospital and the autopsy findings. At autopsy 88 pellets were retrieved from her body. The main pathological findings included pulmonary and cerebral edema. Despite the presence of free heroin in the gastric contents, toxicological analysis did not demonstrate any heroin or its metabolites in blood or tissue extracts. This case illustrates the challenges in postmortem evaluation of narcotic fatalities and the need to consider all factors such as antemortem history, toxicology results and autopsy findings in forensic diagnosis
\end{abstract}

\section{INTRODUCTION}

Body packers ("Mules") are smugglers who conceal narcotic for transport in their bodies. The drugs are usually concealed in the gastro-intestinal tract (GIT) as swallowed sealed package, while in some cases they are placed in the ear, vagina, or even foreskin(1). The package may be wrapped in cellophane, layers of latex, rubber cots, condoms, plastic bags, aluminum foil, plastic foil, wax sealing, carbon paper or self adhesive tape $(2,3)$. The extent of smuggling by body packers is unknown, and little information exists regarding the fatalities or near fatalities that arise from this practice(4). However, it is thought that because of increasing sophistication in intracorporeal concealment methods, only a few body packers die of poisoning because of leakage of an internally concealed container(5). Most reports of the heroin packing have described cocaine smugglers in the United States of America, while a few cases have been documented in young male Africans and Asians traveling in European countries $(1,4,6)$. Heroin is a very expensive and highly potent semi-synthetic opiate, and has been favoured in illicit drug trafficking(4). A single body packer may literally conceal drugs worth over US\$ 100,000(6).

The first autopsy on a narcotic fatality described cerebral and pulmonary congestion in 1852(7). Additional signs, may include portal adenopathy, track marks, renal nephropathy and leukoncephalopathy. These pathological signs are considered "typical" but "neither certain nor characteristic", thus a thorough clinical and decedent history is often more revealing than the autopsy(1-7). Toxicology finding may not be particularly useful, thus in conducting a forensic investigation of death due to narcotism, it is important to consider all factors rather than one or two in isolation $(3,6,7)$.
We present a case of death caused by heroin intoxication in a body packer and highlight the challenges in its forensic diagnosis. We hope that the information herein will contribute to the further understanding of narcotic fatalities in body packers.

\section{CASE REPORT}

The decedent, a 38-year-old African Ugandan woman arrived at the Jomo Kenyatta International Airport (JKIA) in Nairobi from Entebbe, on transit from Karachi to Johannesburg. She was arrested on suspicion by the airport police that she was a drug trafficker (body packer) but details of what arose the suspicion in this care were not available to us. Police usually identify suspects from their intoxication appearance and behaviour, travel route and destination, doubtful details about journey, local contact person, or relevant information given by Interpol before arrival of such a person(2).

She was detained for about 12 hours at the airport; and while in custody she expelled a pellet par anum after being forcefully fed on milk and ugali (prepared maize meal). This is a standard police procedure at JKIA aimed at inducing defecation through the gastro-colic reflex, after which the pellets could be physically sought in the stool. She vomited several times, collapsed thereafter and was taken to a main city hospital in Nairobi.

She was received at the accident and emergency centre (A\&E), unresponsive with no spontaneous respiration or palpable pulses. It took 30 minutes of advanced cardiopulmonary resuscitation and total of $0.8 \mathrm{mg}$ of Naloxone before a sinus rhythm was recorded at 146 beats/minute and a blood pressure (BP) of 139/ $45 \mathrm{mmHg}$. She was then admitted to the intensive care unit comatose with a Glasgow coma scale of $3 / 15$, 
Table 1

Observation at autopsy

Blood stained fluid

Congestion

Haemorrhages

Anatomical location of the pellets
Nostrils, nasopharynx pericardial sac,

pleural cavities, peritoneum

Irides, conjuctivae, nasopharyngeal mucosa, gastric mucosa, colonic mucosa, liver kidneys and celebral vessels

Sub-endocardial surface of the left ventricle,

lungs (haemorrhage consolidations)

Stomach (5 pellets) and colon (83 pellets)
BP $70 / 50 \mathrm{mmHg}$, heart rate $38 / \mathrm{min}$ of Wenckebach pattern and pupil size of $5 \mathrm{~mm}$ bilaterally, equal and non-reacting to light. Coarse crepitations were heard on the chest while the abdomen was distended with firm mobile masses. Chest X-ray was suggestive of pulmonary edema. The pupils remained fixed at dilation and the patient required respiratory support. A low intensity Xray done on the second day revealed opacified foreign bodies in the large gut, nine of which were retrieved manually, after the patient had been given oral Dulcolax and enema. Her BP by the third day was unrecordable despite inotropic support and she went into bradycardia and eventually terminal asystole(8). Toxicological reports on clinical samples at admission indicated that heroin was detected in gastric lavage fluid $(597.70 \mathrm{mg} / \mathrm{L})$ and in blood $(10.58 \mathrm{mg} / \mathrm{L})$.

Autopsy Anatomical Findings: A summary of the gross pathological finding is given in Table 1 . There was minimal rigidity in the upper limbs and jaws. No traces of trauma were evident except for those resulting from iatrogenic injections on the dorsum of the hands and the cubital fossae.

Subcutaneous dissection did not show any needle track marks anywhere on the body. The brain had outstanding features of increased intracranial pressure including flattened gyri, consular herniation and uncal grooving. There was marked congestion of cerebral vessels, but no evidence of intracranial haemorrhage. Coronal and transverse sections of the brain were normal. The stomach contained moderate amount of whitish-yellow pasty material and five intact black pellets. The gastric mucosa was markedly congested with no ulcerations. The duodenum, jejunum, ileum, appendix and caecum were normal. There was no evidence of intestinal obstruction. However, the transverse and sigmoid colon contained whitish-yellow pasty material and 83 pellets, which were retrieved. The pellets were all wrapped in the same material - black adhesive tape, each about the size of walnut, overlying a multilayer of covering of different material. The police handed these over to the Government Chemist for analysis. There were no other significant positive anatomical findings from the autopsy.
Postmortem toxicological findings: Toxicological analyses by gas chromatography were performed on postmortem femoral blood, gastric and duodenal contents, liver and kidney extracts by the Government Chemist Department. A total of $7.4 \mathrm{mg}$ of heroin was obtained from the free gastric content. No heroin or other chemically toxic substances was detected in the blood sample, liver or kidney extracts. The toxicology report also indicated that the whitish-brown powder sealed in the pellets was heroin.

\section{DISCUSSION}

We believe that the decedent died of the effects of acute heroin toxicity that could not be medically reversed. This occurred immediately prior to her admission leading to irreversible pathophysiological changes that caused her to go into coma and resulted in her death; long after the blood and tissues levels had gone down. This is because the blood level of $10.58 \mathrm{mg} / \mathrm{L}$ taken at admission was well above the minimum lethal blood level of heroin at $0.2 \mathrm{~m} / \mathrm{L}(9,10)$. Opiate absorption occurs mostly in the small intestine to reach peak plasma levels after 30-90 minutes (7). The heroin must have been released from one or more of the pellets, while she was undergoing the interrogation, which lasted 12 hours prior to her collapsing.

The main findings at autopsy were pulmonary edema, blood tinged edema fluid in the airways, congestion of the cerebral vessels, hyperemic gastric and colonic mucosa, and subendocardial haemorrhage. These are consistent with pathological finding from similar cases reported of narcotic fatalities $(1,4-7)$. The mechanism of death is usually related to respiratory failure secondary to the direct suppressor effects of the opiate metabolites on respiratory centers leading to hypoxia, hypercapnia and pulmonary edema. The actual pathophysiology of pulmonary edema is unclear, but is thought to arise as a direct result of hypoxia, capillary permeability and fluid extravasations into the alveoli $(7,11,12)$. Sub-endocardial haemorrhage as documented in this case result as a sequele of pulmonary edema as well as injury caused directly by hypoxia and heroin 
toxicity to the coronary capillaries. No specimens were taken for histology, because we did not foresee any additional value from histology in this particular case having been limited by constraints previously explained elsewhere(11). In all, 98 pellets were retrieved from the decedent, one at the airport. Nine while the patient was in hospital and 88 at autopsy.

We question the safety of forcefully feeding heavy meals to body packer suspects. It is possible that such a manoeuver could affect the integrity of the drug pellet, especially if poorly packaged causing rupture and acute toxicity(5). Safer methods for detection of intracorporal concealment have been suggested. X-ray identification of the drug pellets, and if the results of plain X-ray remain unclear in strongly suspicious cases, computer tomography could be used as it shows the best detection capacity $(1,3,14,16)$. Urine testing has been reported by some investigators to be a reliable method for identification of drugs in every type of internal concealment although some investigators have found negative results $(1,15)$. Ultrasonography and saliva screening have been found unsuitable(16).

Failure to demonstrate any heroin in blood at autopsy in this case could be due to the decline in the heroin level documented at the time of acute toxicity, during the three day period when the patient was in the hospital's critical care unit. Tissue re-distribution of heroin and its metabolites is very $\operatorname{rapid}(9,17)$. Once the conversion of heroin to morphine is completed, the measurable detection of the morphine is limited to 12 hours $(7,9,17)$. Thus even with the most sensitive methods the maximum limit of detection does not exceed 48 hours in plasma $(7,17)$. The free heroin in the stomach contents probably leaked from one of the five pellets in the stomach prior to death before it could be absorbed.

In conclusion postmortem pathological signs in body packers; are combined evidence of presence of the drug pellets in the body as well as toxicological analysis in ante-mortem history, and clinical reports. Toxicological analysis of the presence of the drug in body fluids such as plasma and tissue extracts may not be of value when the level of the drug falls after a period of metabolism. We recommend that the force-feeding method of achieving drug pellet expulsion from suspected body packers be abandoned and replaced by safer yet sensitive methods such as radiology and toxicology.

\section{ACKNOWLEDGEMENTS}

To Mathew Lynch and Beng Beng Ong for reading the manuscript

\section{REFERENCES}

1. Heinemann, A.Miyaishi, S., Iwer Sen, S., et al. Bodypacking, as a cause of unexpected sudden death. Forensic Sci. Inter. 1998; 92:1-10

2. Glass, J., and Scott, H. Surgical Mules: The smuggling of drugs in the gastrointestinal tract. J. R. Soc. Med. 1995; 88:450-453.

3. Bogusz, M. J. Althoff, H., Erkens, M. et al. Internally concealed cocaine: Analytical and diagnostic aspects. J. Forensic Sci. 1995; 40:811-815.

4. Wetli, C. and Mittleman, R. The "Body packer syndrome"Toxicity following ingestion of illicit drug packaged for transportation. J. Forensic. Sci. 1981; 264:492-500.

5. Mc Cleave, N. R. Drug smuggling by Body packers. Med. Pathol. 1993; 159: 750-754.

6. Wetli, C. Rao, A., Rao, V.J. et al. Fatal Heroin Body packing. Amer. J. Forensic Med. Pathol. 1997; 18: 312-318.

7 Karch, S. B. The pathology of Drug Abuse 2nd Ed. CRC press, USA, 1996.

8. Olaly, E. Heroin overdose in a "mule". The Nairobi Hospital proceeding. 1999; 3:113-116.

9. Wetli, C. V., and Wright, R. K. Death caused by recreational cocaine use. JAMA 1979; 241:2519-2522.

10. Lepere, B., Gourarier, L., Sanchez, M. et al. Reduction in the number of lethal heroin overdoses in France since 1994. Focus on substitution treatments. Ann. Med. Inter. 2001; 152 Suppl 3:1S5-12.

11. Steentoft, A., Worm, K. and Christensen, H. Morphine concentration in autopsy material from fatal cases after intake of morphine and /or heroin. J. Forens. sci. 1988 28:87-94.

12. Duberstein, J., Adams, G. and Schnoll S. The impact of substance abuse on the respiratory system chest 1986; 89: 266-269.

13. Olumbe, A. K. O. Forensic Medicine in Kenya; a person view. East Afr. Med. J. 2000; 77:60-62.

14. Karhunen, P., Souranja, H., Penttila, A. et al. Pitfalls in the diagnosis of drugs smugglers abdomen. J. Forensic Sci. 1991; 36:397-402.

15. Gheardi, R., Baud, F. Leporc. et al. Detection of drugs in the urine of body -packers. Lancet 1988; 14:1076-1077

16. Mc Carron, M. and Wood, J. The cocaine body packer syndrome: diagnosis and treatment JAMA 1983; 250: 14171420.

17. Sawyer, W., Goedens, D. and Fomey, R. Heroin morphine and hydromorphine determination in postmortem by high perfomance liquid chromatography. J. Forens. Sci. 1988; 33:1146-1155. 